\title{
Update to readers and authors on ethical and scientific misconduct: retraction of the "Boldt articles"
}

\author{
Donald R. Miller, MD
}

Received: 22 June 2011/Accepted: 30 June 2011/Published online: 29 July 2011

(C) Canadian Anesthesiologists' Society 2011

In this issue of the Journal, most regrettably, we publish retraction notices related to five articles authored by Dr. Joachim Boldt et al. which were published during the years 2000-2003. These retraction notices refer to five of 88 articles which appear on the Editors' Joint Statement recently posted on the websites of 18 journals, including the Canadian Journal of Anesthesia. ${ }^{1}$ This unprecedented number of simultaneous article retractions reflects more than a decade of published research examining colloid intravenous solutions and the clinical pharmacology of several anesthetic techniques and drugs used in the perioperative setting. As explained in the Editors' Joint Statement, "the retraction of the articles in the Table for lack of Institutional Review Board (IRB) approval means that the research was unethical, and IRB approval for the research was misrepresented in the published article. It does not mean that the research results per se are fraudulent. Klinikum Ludwigshafen has commissioned an investigating committee to systematically assess the veracity of the findings presented in Dr. Boldt's articles against patient and laboratory records. We will communicate to our readers any finding of data fabrication, falsification, or misrepresentation identified by the investigating committee at Klinikum Ludwigshafen." 1

These articles are retracted as a result of a recent internal investigation at Klinikum Ludwigshafen in Germany where Dr. Boldt worked for over two decades. The investigation began after Dr. Steven Shafer, Editor-in-Chief of

D. R. Miller, MD $(\bowtie)$

Editor-in-Chief, Editorial Office,

Canadian Journal of Anesthesia, c/o Department

of Anesthesia, The Ottawa Hospital, General Campus,

CCW Room 1409, 501 Smyth Road, Ottawa, ON K1H 8L6,

Canada

e-mail: dmiller@toh.on.ca
Anesthesia \& Analgesia, was alerted to concerns from three readers about the implausibility of the very small standard deviations of interleukin IL- 6 concentrations reported in an article published in December 2009. ${ }^{\mathrm{A}}$ As explained by Dr. Shafer in a recent editorial, it required several months to establish the governing body responsible for the ethical conduct of research at Klinikum Ludwigshafen, the hospital in Germany where the study originated. ${ }^{2}$ In another recent editorial, Drs Hoffart, Teichmann, and Wessler explain the rather complex and unique role of the ethics committee and the State Medical Association in Germany which oversees biomedical research in that country. ${ }^{3}$ It was the Landesärztekammer Rheinland-Plafz ("LÄK-RLP"), the State Medical Association of Rheinland-Pfalz, which investigated these concerns. According to Drs Hoffart et al., the LÄK-RLP is authorized to investigate whether a physician has followed the German Code of Deontology or respective laws. ${ }^{3}$ Their investigation determined that Dr. Boldt, who at the time was Chair of the Clinic of Anaesthesiology and Intensive Care at Klinikum Ludwigshafen, had failed to respect the German Code of Deontology. The investigation identified a number of misrepresentations in the article in question, ${ }^{\mathrm{A}}$ including the egregious observation that there were no original patient or laboratory data to support the findings in the study. Furthermore, there was no convincing evidence that the study had actually taken place. In essence, the study published in Anesthesia \& Analgesia in 2009 was fabricated. The article was retracted, ${ }^{4}$ and the state government referred the matter to the German Office of the Public Prosecutor. In late November 2010, the administration at Klinikum Ludwigshafen and

\footnotetext{
$\overline{\mathrm{A}}$ Boldt J, Suttner S, Brosch C, et al. Cardiopulmonary bypass priming using a high dose of a balanced hydroxyethyl starch versus an albumin-based priming strategy. Anesth Analg 2009; 109: 1752-62.
} 
Dr. Boldt agreed that his practice at the Klinikum would be terminated. $^{3}$

Retraction of the fabricated article immediately cast a shadow of doubt regarding all of the work published by Dr. Boldt. In late 2010, two investigating committees were established to determine whether Dr. Boldt and his co-authors adhered to the regulations outlined in German federal laws and the Code of Deontology when they published their articles. The ongoing investigation led by Dr. Wissler has evolved into an extensive review of the ethical conduct of Boldt's published research. Between December 2010 and February 2011, Dr. Steven Shafer assumed the considerable task of coordinating the effort of the Editors-in-Chief of 18 medical journals to forward to the LÄK-RLP a list of articles published by Dr. Boldt and deemed, by the Editors-in-Chief of the respective journals, to contain data which might have been fabricated. This pursuit represented a unique international editorial collaboration. It involved editorial judgement calls taking into consideration the nature of the studies and the similar types of data being reported across studies. In February 2011, the LÄK-RLP delivered its report based on an internal review of over 100 published articles co-authored by Dr. Boldt. In their report, the LÄK-RLP specified that no apparent evidence of Institutional Research Board (IRB) approval existed in 88 studies. When the LÄK-RLP gave Dr. Boldt sufficient opportunity and time to produce evidence of IRB approval for each of the studies in question, he failed to do so. Furthermore, no evidence of IRB approval was provided by any of Dr. Boldt's co-authors. During the investigation, it became clear that misleading statements had been given to indicate that IRB approval had been obtained for the respective published studies.

Unquestionably, conducting research in humans without approval from an IRB or Research Ethics Board (REB) or equivalent is unethical. The studies listed in the Table are hereby retracted from the Canadian Journal of Anesthesia due to lack of any evidence of ethical approval for the studies in question. We recognize the serious implications of article retractions, and recognize that this process often takes some months. At the time of this writing, other journals have either retracted articles listed on the Editors' Joint Statement or are in the process of retracting articles. There are ongoing investigations and our readers will be apprised of any further developments.

Consistent with international editorial policies, all journals' Instructions for Authors should state explicitly that manuscripts describing investigations performed in humans will not be considered for publication unless the study was approved by the authors' IRB or REB. Furthermore, as regards the Journal, a statement concerning REB approval and consent procedures must appear at the beginning of the methods section of each manuscript
Table Articles authored by Joachim Boldt retracted from the Canadian Journal of Anesthesia

Piper SN, Kumle B, Maleck WH, Kiessling AH, Lehmann A, Röhm $K D$, Suttner $S W$, Boldt J. Diltiazem may preserve renal tubular integrity after cardiac surgery. Can J Anesth 2003; 50: 285-92.

Lang K, Suttner S, Boldt J, Kumle B, Nagel D. Volume replacement with HES 130/0.4 may reduce the inflammatory response in patients undergoing major abdominal surgery. Can J Anesth 2003; 50: 1009-16.

Piper SN, Suttner SW, Röhm KD, Maleck WH, Larbig E, Boldt J. Dolasetron, but not metoclopramide prevents nausea and vomiting in patients undergoing laparoscopic cholecystectomy. Can J Anesth 2002; 49: 1021-8.

Piper SN, Fent MT, Röhm KD, Maleck WH, Suttner SW, Boldt J. Urapidil does not prevent postanesthetic shivering: a dose-ranging study. Can J Anesth 2001; 48: 742-7.

Piper SN, Boldt J, Schmidt CC, Maleck WH, Brosch C, Kumle B. Hemodynamics, intramucosal $\mathrm{pH}$ and regulators of circulation during perioperative epidural analgesia. Can J Anesth 2000; 47: $631-7$.

reporting the results of clinical trials or observational studies involving human research. We require written consent. With regard to observational studies, if the REB waived the requirement for consent, then this must be stated explicitly. We have updated our policy more recently and now require the name of the governing REB body (hospital or university) and the month and year of protocol approval to be stated at the beginning of the methods section of the manuscript. We will also archive the contact information of the ethical review board. Any systematic data gathering efforts in patients or volunteers must also be approved by an REB or adhere to duly recognized local/national regulations. Authors may be asked to provide a copy of the REB approval form at the time of article submission. Lack of appropriate documentation will be grounds for rejection.

There is a natural trust relationship between editors and authors; therefore, statements about originality, authorship, and appropriate ethical approval of article submissions involving studies in humans are considered to be valid unless there is compelling evidence to the contrary. Editorial offices do not have the authority, resources, or responsibility to investigate misconduct. That responsibility lies with the institution where the research originated. In this instance, the Editors-in-Chief have no choice but to follow the guidance of LÄK-RLP and Klinikum Ludwigshafen.

The implications of the 88 article retractions will take years to resolve fully. As mentioned at the beginning of this article, much of the related research involves hydroxyethyl starches (HES). There are over 50 published randomized controlled trials evaluating HES 130/0.4. ${ }^{5}$ It is noteworthy that one-third of these trials involving elective 
surgery patients were published by Boldt and co-authors. Despite limitations of many related clinical trials, colloid solutions containing HES 130/0.4 are now used widely for intravenous therapy. In their recent review of hydroxyethyl starches, Drs Reinhart and Takala conclude that "published clinical data are inadequate to support the conclusions that HES 130/0.4 is safer than other HES solutions in surgical and critically ill patients". 6 Thus, additional appropriately conducted clinical trials will be necessary to determine more conclusively the role of these intravenous colloid solutions in clinical practice. While the fallout from these article retractions continues to be analyzed, we await (and will eventually report) further results of the ongoing investigations at Klinikum Ludwigshafen in Germany. The Journal's editorial team remains steadfast in its ongoing commitment to ensure the integrity of the scientific record.

\section{Mise à jour destinée aux lecteurs et aux auteurs concernant la malhonnêteté scientifique et les infractions aux règles d'éthique: la rétractation des articles du Dr Boldt}

Dans ce numéro du Journal, nous regrettons de devoir publier des avis de rétractation liés à cinq articles rédigés par le Dr Joachim Boldt et coll. et publiés en 2002-2003. Ces avis de rétractation concernent cinq des 88 articles cités dans la Déclaration conjointe des rédacteurs récemment affichée sur les sites Internet de 18 revues, y compris celui du Journal canadien d'anesthésie. ${ }^{1} \mathrm{Ce}$ nombre sans précédent de rétractations simultanées d'articles touche plus d'une dizaine d'années de recherches publiées sur les solutions colloïdales intraveineuses et sur la pharmacologie clinique de plusieurs techniques et médicaments d'anesthésie utilisés en cadre périopératoire. Comme l'explique la Déclaration conjointe des rédacteurs, «la rétractation des articles du Tableau en raison de l'absence d'approbation du Comité d'éthique de la recherche (CER) signifie que les recherches n'étaient pas déontologiques, et que l'approbation du CER pour ces recherches a fait l'objet d'une représentation erronée dans l'article publié. Toutefois, cela ne signifie pas que les résultats de l'étude sont, en soi, frauduleux. Le Klinikum Ludwigshafen a mandaté un comité d'enquête afin d'évaluer systématiquement la véracité des données présentées dans les articles de Dr Boldt en les comparant aux dossiers des patients et aux résultats de laboratoire. Nous informerons nos lecteurs de toute information quant à la fabrication, la manipulation ou la représentation erronée de
Tableau Articles de Joachim Boldt rétractés du Journal canadien d'anesthésie

Piper SN, Kumle B, Maleck WH, Kiessling AH, Lehmann A, Röhm $K D$, Suttner $S W$, Boldt J. Diltiazem may preserve renal tubular integrity after cardiac surgery. Can J Anesth 2003; 50: 285-92.

Lang K, Suttner S, Boldt J, Kumle B, Nagel D. Volume replacement with HES 130/0.4 may reduce the inflammatory response in patients undergoing major abdominal surgery. Can J Anesth 2003; 50: 1009-16.

Piper SN, Suttner SW, Röhm KD, Maleck WH, Larbig E, Boldt J. Dolasetron, but not metoclopramide prevents nausea and vomiting in patients undergoing laparoscopic cholecystectomy. Can J Anesth 2002; 49: 1021-8.

Piper SN, Fent MT, Röhm KD, Maleck WH, Suttner SW, Boldt J. Urapidil does not prevent postanesthetic shivering: a dose-ranging study. Can J Anesth 2001; 48: 742-7.

Piper SN, Boldt J, Schmidt CC, Maleck WH, Brosch C, Kumle B. Hemodynamics, intramucosal $\mathrm{pH}$ and regulators of circulation during perioperative epidural analgesia. Can J Anesth 2000; 47: 631-7.

données telles que découvertes par le comité d'enquête du Klinikum Ludwigshafen. ${ }^{1}$

La rétractation de ces articles est le résultat d'une enquête interne réalisée récemment au Klinikum Ludwigshafen en Allemagne, institution où le Dr Boldt a travaillé pendant plus de vingt ans. L'enquête s'est ouverte après que Dr Steven Shafer, rédacteur en chef de la revue Anesthesia \& Analgesia, a eu connaissance d'inquiétudes exprimées par trois lecteurs quant à l'invraisemblance des écarts types très petits des concentrations d'interleukine IL-6 rapportés dans un article publié en décembre 2009. ${ }^{\mathrm{A}}$ Comme l'explique le Dr Shafer dans un éditorial récent, il a fallu plusieurs mois avant de déterminer quel était l'organe directeur responsable de veiller à la réalisation éthique des recherches au Klinikum Ludwigshafen, l'hôpital allemand dans lequel l'étude en question a été réalisée. ${ }^{2}$ Dans un autre éditorial récent, les Drs Hoffart, Teichmann et Wessler expliquent le rôle relativement complexe et unique, en Allemagne, du Comité d'éthique et de l'Association médicale d'état, les deux organismes qui supervisent les recherches biomédicales dans ce pays. $^{3}$ La Landesärztekammer Rheinland-Pfalz ( « LÄK-RLP »), l'Association médicale d'état du Land de Rhénanie-Palatinat, est l'organisme qui a examiné ces questions. Selon les Drs Hoffart et coll., la LÄK-RLP a le droit d'enquêter pour déterminer si un médecin a suivi le Code de déontologie allemand et les lois en vigueur ou non. ${ }^{3}$ Leur enquête a conclu que le Dr Boldt, qui était alors président de la clinique d'anesthésiologie et de soins intensifs au Klinikum Ludwigshafen, n'avait pas respecté

\footnotetext{
A Boldt J, Suttner S, Brosch C, et al. Cardiopulmonary bypass priming using a high dose of a balanced hydroxyethyl starch versus an albumin-based priming strategy. Anesth Analg 2009; 109: 1752-62.
} 
le Code de déontologie allemand. L'enquête a identifié plusieurs représentations erronées dans l'article en question, ${ }^{\mathrm{A}}$ notamment l'observation flagrante qu'il n'y avait aucune donnée originale tirée de dossiers de patients ou de laboratoire pour appuyer les résultats de l'étude. En outre, aucune donnée probante convaincante ne prouvait que l'étude avait en fait été réalisée. En bref, l'étude publiée dans la revue Anesthesia \& Analgesia en 2009 avait été fabriquée de toutes pièces. L'article a été rétracté, ${ }^{3}$ et le gouvernement d'état a renvoyé la question au Parquet allemand. À la fin du mois de novembre 2010, l'administration du Klinikum Ludwigshafen et Dr Boldt sont parvenus à un accord concernant la rupture du contrat de pratique du Dr Boldt à la clinique.

La rétractation de l'article fabriqué a immédiatement jeté une ombre de doute sur tous les travaux publiés par le Dr Boldt. À la fin de l'année 2010, deux comités d'enquête ont été mis sur pied avec pour mission de déterminer si le Dr Boldt et ses co-auteurs avaient respecté les règlements décrits dans les lois fédérales allemandes et le Code de déontologie lors de la publication de leurs articles. L'enquête en cours, menée par le Dr Wessler, s'est transformée en une évaluation minutieuse de la conduite éthique des recherches publiées par le Dr Boldt. Entre décembre 2010 et février 2011, le Dr Steven Shafer a assumé la tâche substantielle de coordonner les efforts des rédacteurs en chef de 18 revues médicales afin de faire parvenir à la LÄK-RLP une liste des articles publiés par le Dr Boldt qui, selon les rédacteurs en chef des revues respectives, pourraient contenir des données potentiellement fabriquées. Cette entreprise illustre une collaboration unique entre les équipes de rédaction. Il a fallu faire preuve de jugement et tenir compte de la nature des études et des types de données semblables rapportés dans différentes études. En février 2011, la LÄK-RLP a livré un rapport se fondant sur l'examen interne de plus de 100 articles publiés et dont la copaternité était imputée au Dr Boldt. Dans ce document, la LÄK-RLP a spécifié qu'il n'existait aucune donnée apparente d'approbation du Comité d'éthique de la recherche (CER) pour 88 études. Lorsque la LÄK-RLP a donné au Dr Boldt l'occasion et le temps nécessaires à présenter les preuves d'une approbation du CER pour chacune des études en question, il ne l'a pas fait. En outre, aucune preuve d'approbation du CER n'a été fournie par quiconque des co-auteurs du Dr Boldt. Au cours de l'enquête, il est devenu évident que des déclarations mensongères avaient été faites à l'effet qu'une approbation du CER avait été obtenue pour les études publiées.

Il ne fait aucun doute que la réalisation de recherches chez l'humain sans l'approbation d'un Comité d'éthique indépendant (CEI), d'un Comité d'éthique de la recherche (CER) ou de tout autre organisme équivalent, est contraire à l'éthique. Les études énumérées dans le Tableau sont par conséquent rétractées du Journal canadien d'anesthésie en raison de l'absence de toute preuve certifiant l'obtention d'une approbation du Comité d'éthique pour les études en question. Nous sommes conscients des implications graves liées à la rétractation d'articles, et que ce processus prend souvent plusieurs mois. Au moment de rédiger cet article, d'autres revues ont soit rétracté les articles cités dans la Déclaration conjointe des rédacteurs, soit s'apprêtent à le faire. L'enquête se poursuit et nous informerons nos lecteurs de tout développement futur.

Conformément aux politiques rédactionnelles internationales, toutes les Directives des revues aux auteurs doivent énoncer de façon explicite que les manuscrits décrivant des recherches menées chez l'humain ne seront pas pris en considération pour publication à moins que l'étude n'ait reçu l'approbation du CER ou du CEI des auteurs. En outre, dans le cas du Journal, une déclaration portant sur l'approbation du CER et les processus d'obtention du consentement des patients doit apparaître au début de la section Méthode de chaque manuscrit rapportant les résultats d'études cliniques ou d'études observationnelles impliquant des recherches chez l'humain. Le consentement écrit est requis. En ce qui concerne les études observationnelles, si le CER a renoncé à cette exigence de consentement, cela doit être explicitement mentionné. Nous avons récemment mis à jour notre politique et exigeons désormais que le nom de l'organisme du CER (hôpital ou université) ainsi que la date (mois et année) d'approbation du protocole soient mentionnés au début de la section Méthode du manuscrit. Aussi, nous conserverons les coordonnées du comité d'éthique de la recherche. Toute collecte systématique de données chez des patients ou volontaires devra également avoir été approuvée par un CER ou se conformer à la réglementation locale/nationale. Dans certains cas, les auteurs peuvent être priés de fournir un exemplaire du formulaire d'approbation du CER au moment de la soumission de leur article. L'absence de documentation adéquate peut justifier le rejet du manuscrit.

Il existe une relation naturelle de confiance entre les rédacteurs et les auteurs; pour cette raison, les déclarations concernant l'originalité, la paternité et l'approbation déontologique qui sont requises dans le cas des articles soumis décrivant des études chez l'humain sont prises comme étant valables, sauf si des données convaincantes indiquent le contraire. Les bureaux de rédaction ne disposent pas de l'autorité et des ressources pour examiner les cas de malhonnêteté et n'en ont pas la responsabilité. Cette responsabilité incombe à l'institution d'où provient la recherche. Dans le cas décrit ici, les rédacteurs en chef n'ont d'autre choix que de suivre les recommandations de la LÄK-RLP et du Klinikum Ludwigshafen.

La rétractation des 88 articles va avoir des répercussions qui prendront plusieurs années avant d'être complètement 
résolues. Comme nous l'avons mentionné au début de cet article, beaucoup de ces recherches portent sur l'hydroxyéthylamidon (HES). Plus de 50 études randomisées contrôlées publiées évaluent le HES 130/0.4. ${ }^{5}$ Il est important de souligner qu'un tiers de ces articles qui examinaient des patients de chirurgie non urgente a été publié par le Dr Boldt et ses co-auteurs. Malgré les limites de plusieurs des études cliniques liées, les solutions colloïdales contenant du HES 130/0.4 sont désormais couramment utilisées pour les traitements intraveineux. Dans leur compte-rendu récent sur l'hydroxyéthylamidon, les Drs Reinhart et Takala concluaient que « les données cliniques publiées ne suffisent pas à conclure que le HES 130/0.4 est plus sécuritaire que les autres solutions de HES chez les patients de chirurgie et de soins intensifs ». ${ }^{6}$ Pour cette raison, des études cliniques supplémentaires réalisées dans les règles de l'art seront nécessaires afin de déterminer de façon plus concluante le rôle de ces solutions colloïdales intraveineuses dans la pratique clinique. Alors que les conséquences de ces rétractations d'articles continuent d'être analysées, nous attendons les résultats des enquêtes en cours au Klinikum Ludwigshafen en Allemagne, et nous vous en ferons part. La position de l'équipe de rédaction du Journal demeure constante dans son engagement continu afin de garantir l'intégrité des publications scientifiques.

Competing interests None declared.

\section{References}

1. EIC Joint Statement on Retractions. Editors-in-Chief joint statement regarding published clinical trials without IRB approval by Joachim Boldt. Available from URL: http://www.springer.com/ medicine/anesthesiology/journal/12630 (accessed June 2011).

2. Shafer SL. Shadow of doubt (editorial). Anesth Analg 2011; 112: 498-500.

3. Hoffart J, Teichmann A, Wessler I. Biomedical research in Germany: the role of ethics committee and State Medical Association (editorial). Anesth Analg 2011; 112: 501-3.

4. Shafer SL. Notice of retraction. Anesth Analg 2009; 108: 1953.

5. Hartog CS, Kohl M, Reinhart K. A systematic review of third generation hydroxyethyl starch (HES 130/0.4) in resuscitation: safety not adequately addressed. Anesth Analg 2011; 112: 635-45.

6. Reinhart K, Takala J. Hydroxyethyl starches: what do we still know? Anesth Analg 2011; 112: 507-11. 